\title{
Morphological Analysis of Amoeboid-Mesenchymal Transition Plasticity After Low and High LET Radiation on Migrating and Invading Pancreatic Cancer Cells
}

\author{
ANGELICA FACOETTI ${ }^{1}$, CARMELA DI GIOIA ${ }^{1}$, FRANCESCA PASI ${ }^{2}$, \\ RICCARDO DI LIBERTO ${ }^{4}$, FRANCO CORBELLA ${ }^{2}$, ROSANNA NANO ${ }^{3}$, \\ MARIO CIOCCA ${ }^{1}$, FRANCESCA VALVO $^{1}$ and ROBERTO ORECCHIA ${ }^{1,5}$ \\ ${ }^{1}$ Fondazione CNAO, Pavia, Italy; \\ ${ }^{2}$ Oncological Radiotherapy Unit, IRCCS Fondazione Policlinico San Matteo, Pavia, Italy; \\ ${ }^{3}$ Department of Biology and Biotechnology "L. Spallanzani”, University of Pavia, Pavia, Italy; \\ ${ }^{4}$ Department of Medical Physics, IRCCS Fondazione Policlinico San Matteo Foundation, Pavia, Italy; \\ ${ }^{5}$ University of Milan and Istituto Europeo di Oncologia, Milan, Italy
}

\begin{abstract}
Background/Aim: Cell migration and invasion are fundamental components of tumor cell metastasis that represent the biggest threat to the survival and quality of life of cancer patients. There is clear evidence that ionizing radiation can differently modulate migration and invasiveness of cancer cells depending on the cell lines, the doses and the radiation types investigated. This suggests that motile cells are able to adopt different migration strategies according to their molecular characteristics and external signals. Materials and Methods: In this study, a morphological analysis was performed on pancreatic cancer Aspc-1 cells to evaluate the amoeboid-mesenchymal mobility transition in several experimental conditions considering the role played by factors released by normal and tumor cells, in basal conditions and after low and high Linear Energy Transfer (LET) irradiation. Results and Conclusion: The migratory behavior of Aspc-1 cells is modulated by factors released by normal fibroblasts and tumor cells, and this is in turn modulated by both the radiation dose and the radiation quality.
\end{abstract}

Single cell migration and invasion play pivotal roles in cancer cell invasion and metastasis which are essentially responsible for the dismal prognoses of a majority of cancer patients.

During migration tumor cells show a great variety of morphological changes, even simultaneously (1-3), and the modalities of single-cell migration can be subdivided into

Correspondence to: Facoetti A, Fondazione CNAO, Strada Campeggi 53, Pavia, Italy. Tel: +39 0382078455, Fax: +390382 078903,e-mail: Facoetti@cnao.it

Key Words: Migration, invasiveness, mesenchymal, amoeboid, transition, carbon ions, irradiation, pancreatic cancer, normal fibroblasts. amoeboid- and mesenchymal-type. Cells moving in the mesenchymal mode are elongated, and their movement depends on the proteolytic activity of matrix metalloproteinases (MMP), which permits penetration of the extracellular matrix (ECM). Cells in the amoeboid mode are round with bleb-like protrusions and their movement is mediated by actomyosin contraction, that is regulated by Rhokinase (ROCK). Some cell lines derived from tumors show both these modes of movement. Cells can shift between these two modes also depending on the environmental conditions $(4,5)$.

Suppression or enhancement of the activity of specific molecular pathways determines plasticity of tumor cell migration, allowing to rapidly switch from one type of invasiveness to the other (mesenchymal-amoeboid transition - MAT or amoeboid-mesenchymal transition AMT). Thus, analysis of the morphological appearance during migration may provide useful information on the cell migration process itself and the influence of the tumor microenvironment on this process. Indeed, growth factors and cytokines released by normal and tumor cells represent central extracellular triggers which control these migration programs and thus regulate adaptive invasion strategies. These processes are based on a number of biological characteristics associated with various molecular changes involving proteinases, adhesion molecules, and cell motility factors (6).

In the last years, several studies have described modulation of tumor cell migration and invasiveness after low and high Linear Energy Transfer (LET) irradiation via induction of proteases (4, 7-10). A comprehensive evaluation of the tumor-stromal conditions that may trigger MAT/AMT could help us elucidate the mechanisms of plasticity in the migration strategies of tumor cells after radiotherapy. 
In the present study we, therefore, investigated the morphological plasticity of pancreatic cancer cells migration and invasion in response to different doses of low (photons) and high (carbon ions) LET radiations under well-defined in vitro conditions aiming to mimic the tumor microenvironment.

\section{Materials and Methods}

Cell cultures. Human pancreatic cancer Aspc-1 cells were purchased from Istituto Zooprofilattico Sperimentale della Lombardia e dell'Emilia Romagna (IZSLER, Italy) and cultured in RPMI 1640 medium containing glutamine and supplemented with $100-\mathrm{U} / \mathrm{ml}$ penicillin and streptomycin, $1 \%$ of sodium pyruvate and $20 \%$ of fetal bovine serum (FBS). Human fibroblasts AG01522 were cultured in Minimum essential medium (MEM) alphamodified supplemented with $2 \mathrm{mmol} / \mathrm{l} \mathrm{L}$-glutamine, $1 \%$ penicillin and streptomycin and $20 \%$ of FBS. Because human fibroblasts senesced, they were used at early passage numbers (passages 1118). All cells were grown at $37^{\circ} \mathrm{C}$ in a humidified atmosphere containing $5 \% \mathrm{CO}_{2}$ and splitted using $10 \%$ trypsin when confluent. All media, supplements and chemicals were purchased from Sigma-Aldrich (St. Louis, MO, USA).

Conditioned medium used as chemoattractant in the lower compartments was collected from flasks $24 \mathrm{~h}$ after radiation and filtered with $0.2 \mu \mathrm{m}$ cellulose filters.

Irradiation. Aspc-1 cells and AG01522 cells were irradiated with 6 MV X-rays with a Siemens Primus linear accelerator $(2 \mathrm{~Gy} / \mathrm{min}$ as dose rate) at the Oncological Radiotherapy Department at San Matteo Hospital in Pavia. Cells were grown in T25 flasks and horizontally irradiated at $0 \mathrm{~Gy}, 0.5 \mathrm{~Gy}, 1 \mathrm{~Gy}, 2 \mathrm{~Gy}$ or $4 \mathrm{~Gy}$. Carbonions irradiations were performed at CNAO synchrotron facility in Pavia with a dose rate of $1.39 \mathrm{~Gy} / \mathrm{min} / \mathrm{cm}^{2}$, using scanning beams and same doses used for photons. Cells were vertically irradiated in fulfilled T12.5 flasks in a water phantom at the depth of middle Spread-Out Bragg Peak (energy range=246 $-312 \mathrm{MeV} / \mathrm{u}$ ).

Transwell migration assay. Irradiated or control Aspc-1 cells were harvested and $2.5 \times 10^{5}$ cells suspended in $0 \%$ FBS medium were seeded in duplets on inserts. In the lower compartments $1.5 \mathrm{ml}$ of $20 \%$ fresh complete medium was added. In case of the transwell migration assay to test migration induced by factors released by irradiated pancreatic cancer cells, $1.5 \mathrm{ml}$ conditioned medium collected from irradiated Aspc-1 cells was placed in the lower well as a chemoattractant. Transwell migration assay to test migration morphology induced by irradiated fibroblasts was performed by seeding irradiated Aspc-1 cells in the upper compartment and irradiated AG01522 cells in the lower compartment. AG01522 cells were harvested after radiation and $5 \times 10^{4}$ cells were seeded in the lower compartment of the transwell plate. After $8 \mathrm{~h}$, cells were attached on the bottom of the compartment and inserts with $2.5 \times 10^{5}$ irradiated Aspc- 1 cells of the corresponding dose were added.

All plates tested for migration assay were left in the incubator for $48 \mathrm{~h}$. Then cells on the upper surface of the insert were removed by scrubbing with a cotton tipped swab. Invading cells in the lower surface of the membrane were washed twice with PBS, fixed with $70 \%$ cold ethanol for $3 \mathrm{~min}$, washed and stained for $10 \mathrm{~min}$ with Gentian Violet solution.
Matrigel Invasion assay. Corning BioCoat Matrigel invasion chambers with matrigel coated porous filters $(8 \mu \mathrm{m})$ were used in a transwell assay to test the invasive morphology of irradiated Aspc1 cells in the same conditions described for the migration assays.

Morphological analysis. Five random fields (400x) from each well were photographed, and the proportion of mesenchymal to amoeboid morphology was assessed by visual blinded analysis by two independent observers. Cell morphology was scored as amoeboid type (spherical morphology with no apparent cellular protrusions) or mesenchymal type (spindle-shaped) (4), and the percentage of cells of each morphological type was determined.

Statistical analysis. Data were analyzed by Student $t$-test using GraphPad software. The mean \pm s.d. was determined with a significance level of $p<0.05$.

\section{Results}

In our culturing conditions Aspc-1 cells were morphologically heterogeneous, with a strong preponderance of mesenchymal phenotypes compared to the amoeboid ones.

As a first approach, we scored the number of mesenchymal and amoeboid patterns in low and high LET irradiated Aspc1 cells that migrated towards FBS-enriched medium (Figure 1). Irradiation induced significative changes in the amoeboid/mesenchymal ratio compared to control conditions (not irradiated Aspc-1 cells). In particular, photon radiation determined an increase in amoeboid-mesenchymal transition that was maximum after $0.5 \mathrm{~Gy}: 11.6 \%$ of irradiated migrated cells showing mesenchymal type of motility, compared to $4.7 \%$ of non-irradiated cells. The number of mesenchymal-like migrating cells was slightly increased also with higher doses. Also, carbon ion irradiation determined an increase in mesenchymal motility in the irradiated Aspc-1 cells: this switch towards AMT is correlated with the increase of the dose reaching the maximum value of $18.9 \%$ of Aspc- 1 cells with a mesenchymal-type motion after $4 \mathrm{~Gy}$ of carbon ions.

Subsequently, to better simulate a condition where irradiated cancer cells and irradiated surrounding fibroblasts influence each other in a sustained crosstalk following treatment, irradiated or not irradiated AG01522 cells were seeded in the lower compartment and the mesenchymal- and amoeboid-like motility of accordingly irradiated Aspc- 1 cells was scored (Figure 2). Interestingly, comparing the scoring in the control conditions presented in graphs of Figure 1 and Figure 2, the presence of unirradiated fibroblasts did not influence the modality of migration of unirradiated pancreatic cancer cells. Considering photon radiation, the presence of irradiated fibroblasts in the lower compartment determined a more evident increase in mesenchymal-like mode of single-cell migration in irradiated Aspc-1 cells which was correlated with the radiation dose. A similar trend was observed with carbon ion radiation, although the effect was higher: in the presence of 4 Gy-irradiated normal 


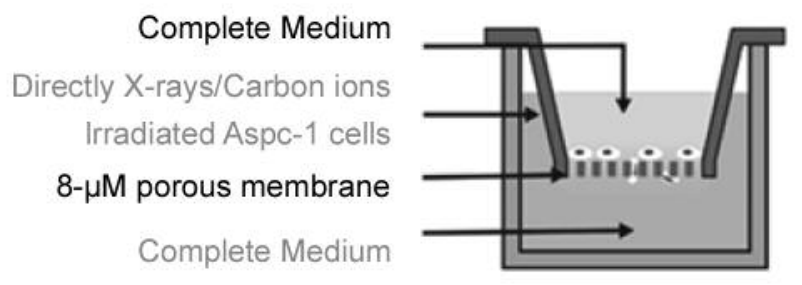

A
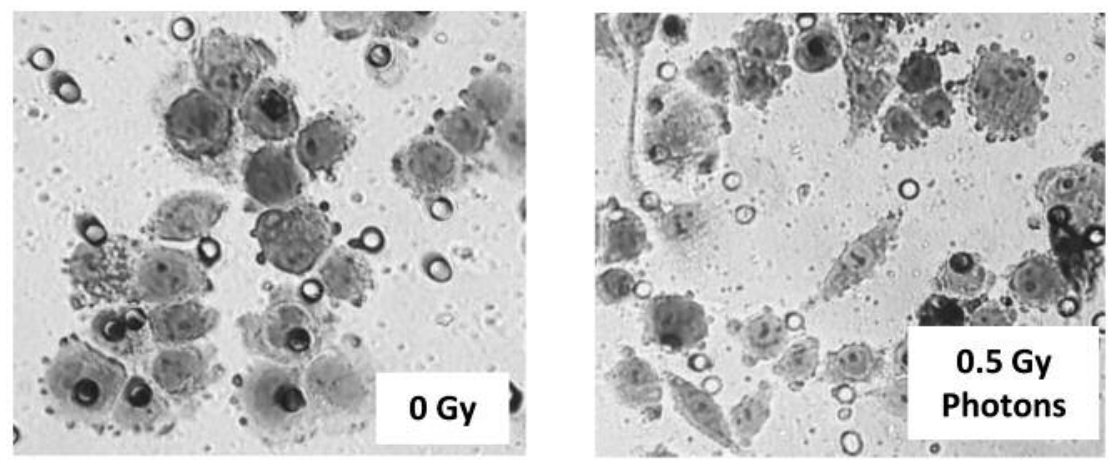

B

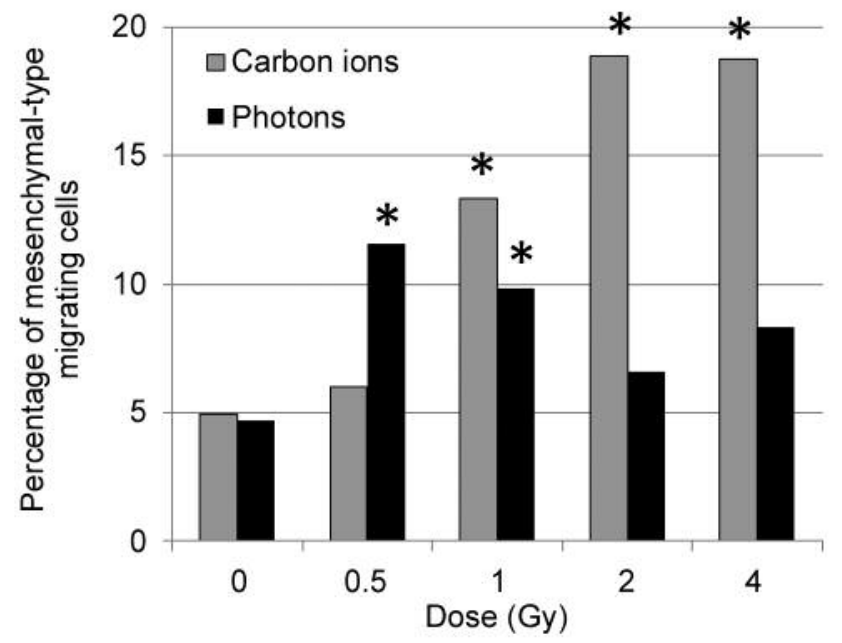

Figure 1. Effects of X-ray or Carbon ion irradiation on the migration morphology of Aspc-1 cells. Irradiated Aspc-1 cells were seeded in the upper well in 0\% FBS medium. Medium with 20\% FBS was used as a chemoattractant (A). Migrated cells were stained with gentian violet and scored for mesenchymal- or amoeboid-morphology, $800 \times(B)$. The percentage of mesenchymal-type migrating Aspc-1 cells was calculated for each dose and radiation type tested $(C)$. Each value represents the mean $\pm S D$ of triplicate experiments. ${ }^{*}$ Statistically significant difference compared to 0 -Gy condition $(p<0.05)$.

fibroblasts $35,9 \%$ of mesenchymal-like pattern of migration was scored in 4 Gy-irradiated Aspc-1 cells.

Finally, in order to better evaluate the role the tumor microenvironment secreted factors played on migration modality we used as a chemoattractant for the irradiated pancreatic cancer cells the conditioned medium collected from low and high LET irradiated Aspe-1 cells (Figure 3).

Concerning photon radiation, the highest percentage $(11.4 \%)$ of mesenchymal-type morphology was scored in
0.5 Gy irradiated Aspc-1 cells migrating towards the medium collected from 0.5 Gy-irradiated Aspc- 1 cells. No significant increases in mesenchymal patterns were observed with the other doses of photons. We observed a peak $(20.6 \%)$ in AMT transition also with carbon ion radiation, although with this type of radiation the dose was 1 Gy. Also, doses higher than 1 Gy of carbon ions determined a significant increase in mesenchymal-like motility compared to 0 and $0.5 \mathrm{~Gy}$. 

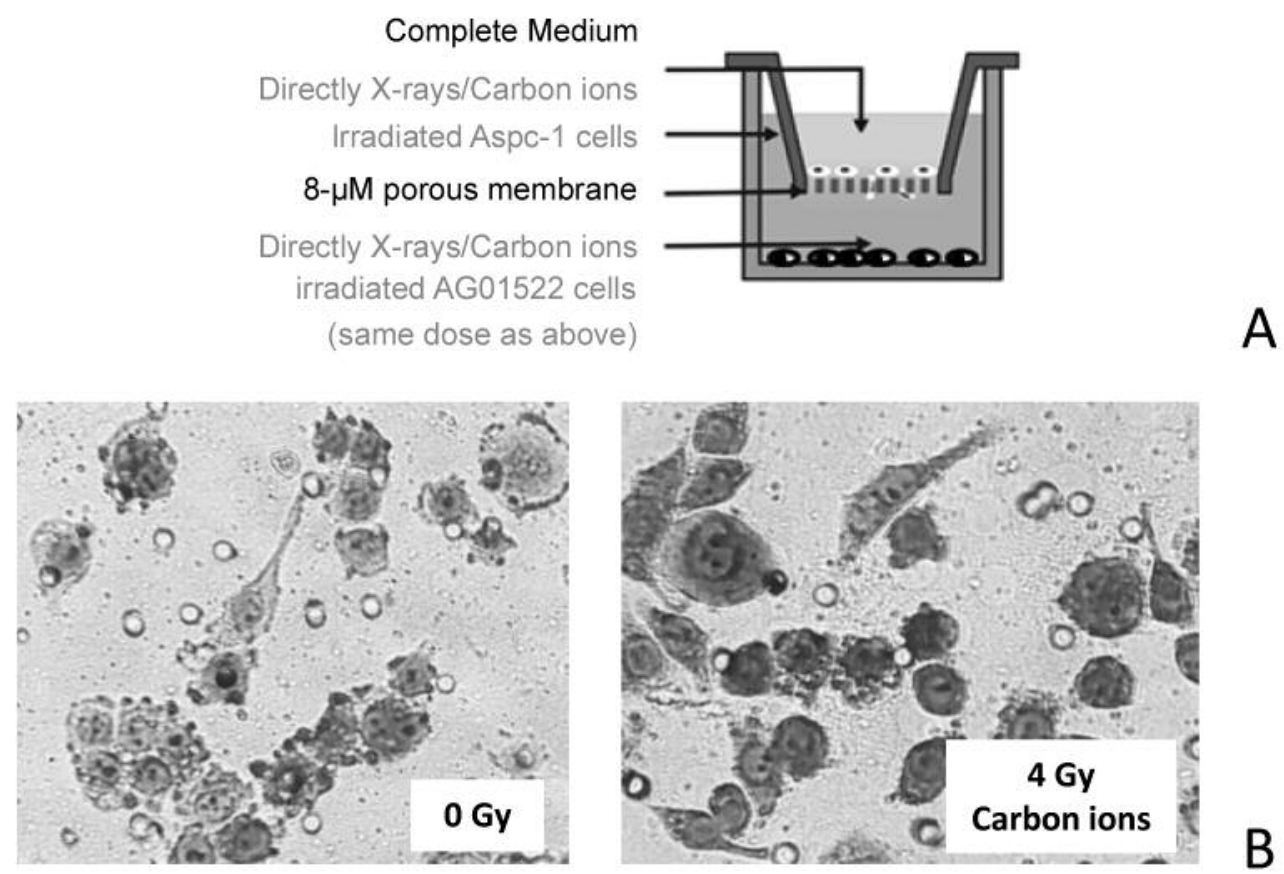

B

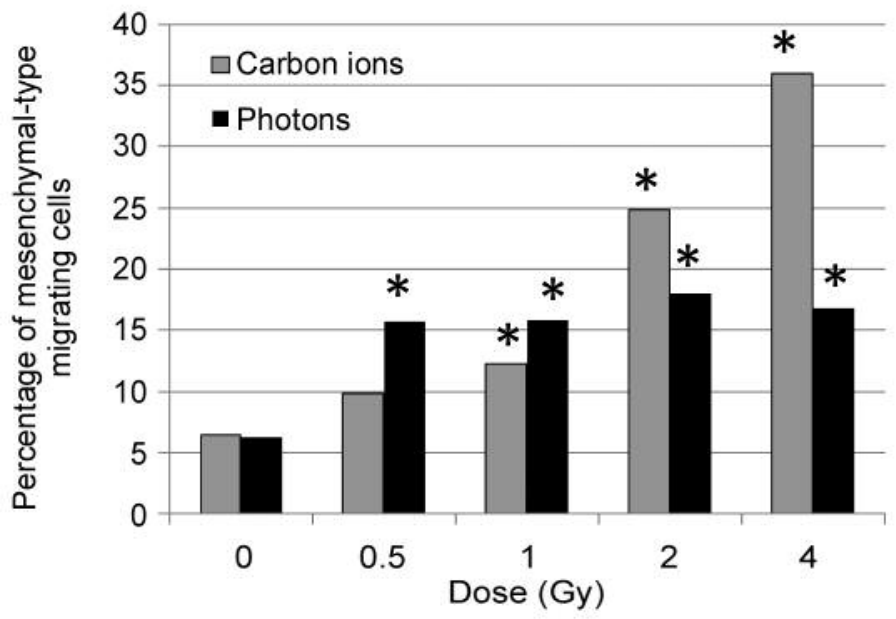

Figure 2. Effects of X-ray or Carbon ion irradiation on the migration morphology of Aspc-1 cells co-cultured with irradiated normal fibroblasts. Irradiated Aspc-1 cells were seeded in the upper well and same-dose, same-radiation-type irradiated AG01522 cells in the lower well (A). Migrated cells were stained with gentian violet and scored for mesenchymal- or amoeboid-morphology 800× (B) The percentage of mesenchymal-type migrating Aspc-1 cells was calculated for each dose and radiation type tested $(C)$. Each value represents the mean $\pm S D$ of triplicate experiments. * Statistically significant difference compared to 0 -Gy condition $(p<0.05)$.

The analysis of invaded Aspc-1 cells toward FBS enriched medium revealed that almost the totality of these cells, either irradiated or not-irradiated, either photons or carbon ions, shows an amoeboid-like motility (between 0 and $5.5 \%$ of cells with mesenchymal appearance with all doses tested and the two radiation types). In parallel, the motility of irradiated pancreatic cancer cells that invaded the matrigel coated membrane toward non-irradiated AG01522 normal fibroblasts was scored along with the mesenchymal pattern. A dramatic switch to mesenchymal-type motility of carbon ion irradiated Aspc-1 cells migrating toward carbon ion irradiated normal fibroblasts. In particular, with the maximum dose tested, i.e. $4 \mathrm{~Gy}$, the mesenchymal cell motility reached $48.2 \%$ of the scored cells and $29.4 \%$ after 1 Gy of carbon ion irradiated Aspc-1 cells and AG01522 (Figure 4). No AMT was observed in pancreatic cancer 


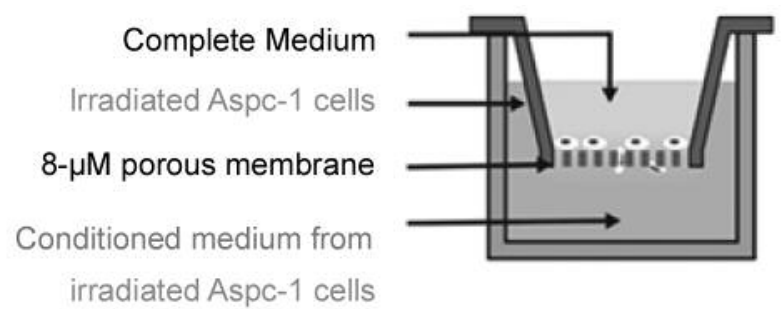

A
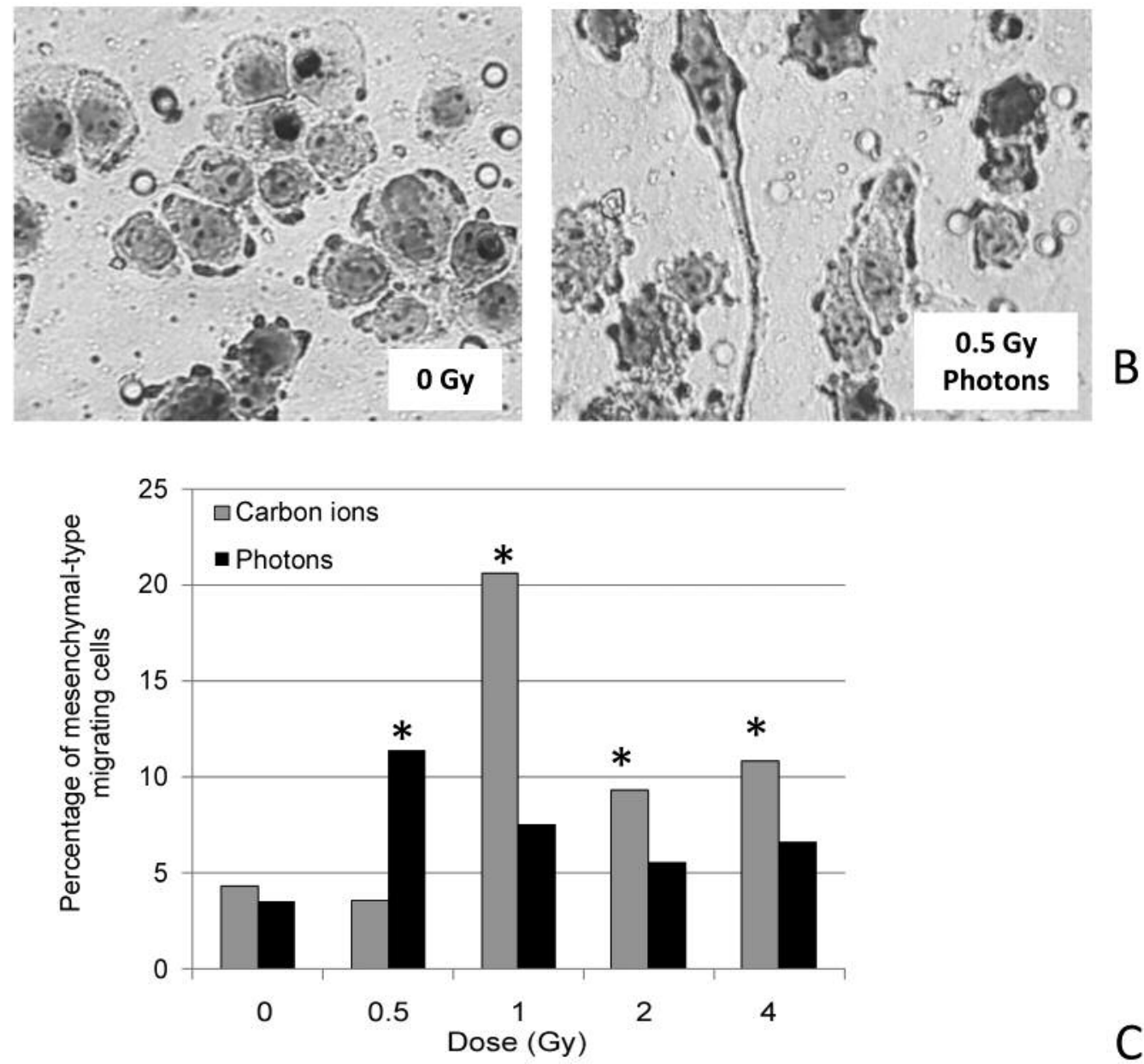

Figure 3. Effects of secreted factors released by irradiated pancreatic cancer cells on the morphology of migrated Aspc-1 cells. Irradiated Aspc-1 cells were seeded in the upper well and medium collected from same-dose, same-radiation-type irradiated Aspc-1 cells used as a chemoattractant (A). Migrated cells were stained with gentian violet and scored for mesenchymal-or amoeboid-morphology, 800x (B) The percentage of mesenchymal-type migrating Aspc-1 cells was calculated for each dose and radiation type tested $(C)$. Each value represents the mean $\pm S D$ of triplicate experiments. *Statistically significant difference compared to 0 -Gy condition $(p<0.05)$.

invaded cells in case of photon radiation of both Aspc-1 and AG01522 cells (data not shown).

\section{Discussion}

Several in vitro and in vivo studies have reported that irradiation may subsequently alter tumor aggression by reducing or promoting the migration and/or invasiveness of the remaining cancer cells, which appear to differ depending on the form and dose of radiation, as well as the investigated cell line $(7,11)$. During the last few years, significant attention has been given to the ability of individual cells to switch between different motility modes, in particular between mesenchymal and amoeboid mobility, and vice 


\section{Complete Medium \\ Directly X-rays/Carbon ions \\ Irradiated Aspc-1 cells \\ 8- $\mu \mathrm{M}$ Matrigel coated porous filters \\ Directly X-rays/Carbon ions irradiated AG01522 cells

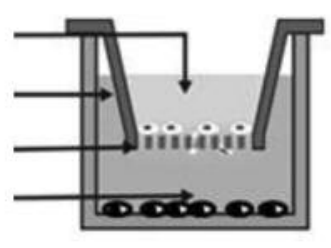 \\ A \\ (same dose as above)}

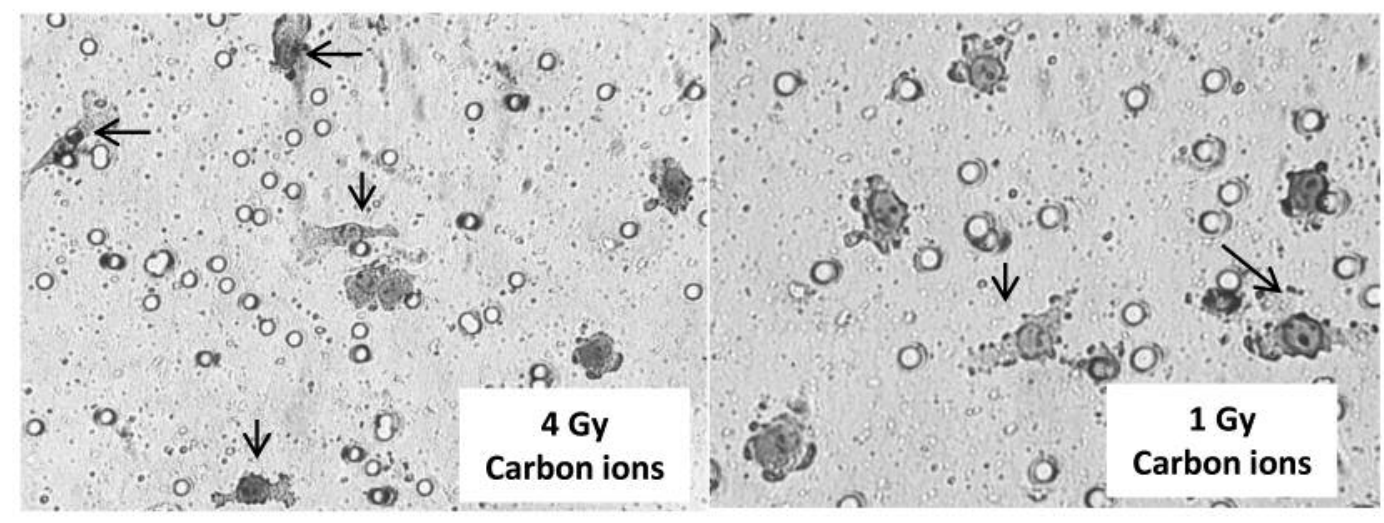

B

Figure 4. Effects of X-ray or Carbon ion irradiation on the morphology of invaded Aspc-1 cells co-cultured with irradiated normal fibroblasts. Irradiated Aspc-1 cells were seeded in the upper well with matrigel coated porous membrane and same-dose, same-radiation-type irradiated AG01522 cells in the lower well (A). Invaded Aspc-1 cells were stained with gentian violet and scored for mesenchymal-or amoeboid-morphology $400 \times(B)$. Arrows indicate mesenchymal-like migration morphologies.

versa (12-18). Cells moving in the mesenchymal mode have elongated and spindle shaped morphologies, and their movement depends on the proteolytic activity of MMPs, which permits penetration of the ECM. Cells in the amoeboid mode are largely rounded with bleb-like protrusions (blebby amoeboid migration) and their movement is mediated by actomyosin contraction, which is regulated by ROCK and is independent from ECM proteolytic degradation (4). Most tumor cells use both cell migration modalities in an adaptive manner (5): changes in migratory strategies can occur rapidly as a form of reply and adaptation by tumor cells to microenvironmental property alterations and intracellular signaling events $(15,16)$.

One very detailed study by Fujita and colleagues investigated the influence of low LET radiation on amoeboid motility of MIAPaCa-2 pancreatic cancer cells (4). In basal conditions they observed a slight induction of AMT after Xray radiation. Nevertheless, the role of secreted factors released by, irradiated or unirradiated, normal and tumor cells and how they cooperate to trigger these migration modalities has not been investigated properly. The present study evaluated the effects of the tumor microenvironment signals on the amoeboid-mesenchymal transition plasticity of migrating and invading Aspc-1 cells with regard to radiation dose and type.
Our results collected in basal conditions, i.e. low or high LET-irradiated cancer cells migrating toward FBS enriched medium, showed a switch of Aspc-1 cells to mesenchymal motility after radiation exposure with important differences according to the radiation type. While the mesenchymal transition proved to be maximal after 2 and 4 Gy of carbon ions, the exposure to photons determined the highest level of cells with mesenchymal morphology after the lowest dose tested (i.e. 0.5 Gy) and then decreased with the higher doses, however remaining above the control values. These data collected with low LET radiations are in agreement with the single study published on irradiated pancreatic cancer cells migration modalities so far (4) where their morphological analysis showed a slight increase in the number of migrating mesenchymal type cells in X-rays-irradiated (2 and 4 Gy) MIAPaCa-2 cells.

To exploit the relevance of interactions between pancreatic cancer cells and surrounding stromal fibroblasts on the migration modality, we scored the amoeboid and mesenchymal migrated Aspc-1 cells cocultured with either irradiated or unirradiated, low and high LET, AG01522 normal fibroblasts. Interestingly the presence of unirradiated fibroblasts did not influence the modality of migration of unirradiated Aspc-1 cells. Ohuchida and colleagues in 2004 investigated the invasiveness of pancreatic cancer cells in response to coculture 
with gamma-ray irradiated fibroblasts (19). They observed that coculture with non-irradiated fibroblasts significantly increased the invasive ability of pancreatic cancer cells and this was further accelerated when cocultured with irradiated fibroblasts. Our study also demonstrates that the ATM of pancreatic cancer cells is increased with the presence of cocultured irradiated fibroblasts. Indeed, the presence of cocultured carbon ion irradiated fibroblasts causes an up to seven-fold increase in the percentage of mesenchymal-type cells in carbon ion irradiated cancer cells compared to control conditions. Also, photonirradiated fibroblasts increased the transition toward mesenchymal morphology however this was not dosecorrelated reaching a sort of plateau level already after $0.5 \mathrm{~Gy}$. Analysis of the invasiveness have highlighted that Aspc- 1 cells invading the matrigel membrane have almost exclusively amoeboid morphology but, surprisingly, the presence of cocultured carbon ion irradiated fibroblasts determines a dramatic switch towards the mesenchymal morphology.

These peculiar differences correlated with the radiation type confirm the different biological mechanisms of response of cells irradiated with either low or high LET radiations.

In conclusion, our present results demonstrate, for the first time, that the migratory and invasive behavior of Aspc-1 cells is modulated by the factors released by normal fibroblasts and tumor cells and this is in turn modulated by both the radiation dose and the radiation quality. These data are of particular importance also in the context of the radiation-induced bystander effects, where even low radiation doses can induce significant effects on normal and cancer neighboring cells through the release of extracellular signals. Surely further studies are necessary to elucidate the molecular mechanisms related to the modulation of pancreatic cancer cells motility by radiation quality and by cell-released factors.

\section{Acknowledgements}

This work was partially founded by INFN project ETHICS.

\section{References}

1 Friedl $\mathrm{P}$ and Wolf $\mathrm{K}$ : Tumour-cell invasion and migration: diversity and escape mechanisms. Nat Rev Cancer 3: 362-367, 2003.

2 Debeir O, Adanja I, Kiss R and Decaestecker C: Models of cancer cell migration and cellular imaging and analysis. In: The Motile Actin System in Health and Disease. Lambrechts A and Ampe C (eds.). Transworld Research Network, Kerala, India, pp. 123-156, 2008.

3 Yamazaki D, Kurisu S and Takenawa T: Regulation of cancer cell motility through actin reorganization. Cancer Sci 96: 379386, 2005.

4 Fujita M, Otsuka Y, Yamada S, Iwakawa M and Imai T: X-ray irradiation and Rho-kinase inhibitor additively induce invasiveness of the cells of the pancreatic cancer line, MIAPaCa-2, which exhibits mesenchymal and amoeboid motility. Cancer Sci 102: 792-798, 2011.

5 Odenthal J, Takes R and Friedl P: Plasticity of tumor cell invasion: governance by growth factors and cytokines. Carcinogenesis 37: 1117-1128, 2016.

6 Ogata T, Teshima T, Kagawa K, Hishikawa Y, Takahashi Y, Kawaguchi A, Suzumoto Y, Nojima K, Furusawa Y and Matsuura N: Particle irradiation suppresses metastatic potential of cancer cells. Cancer Res 65: 113-120, 2005.

7 Goetze K, Scholz M, Taucher-Scholz G and Mueller-Klieser W: The impact of conventional and heavy ion irradiation on tumor cell migration in vitro. Int J Radiat Biol 83: 889-896, 2007.

8 Jung JW, Hwang SY, Hwang JS, Oh ES, Park S and Han IO: Ionising radiation induces changes associated with epithelialmesenchymal transdifferentiation and increased cell motility of A549 lung epithelial cells. Eur J Cancer 43: 1214-1224, 2007.

9 Moncharmont C, Levy A, Guy JB, Falk AT, Guilbert M, Trone JC, Alphonse G, Gilormini M, Ardail D, Toillon RA, RodriguezLafrasse C and Magné N: Radiation-enhanced cell migration/ invasion process: a review. Crit Rev Oncol Hematol 92: 133$142,2014$.

10 Fujita M, Otsuka Y, Imadome K, Endo S, Yamada S and Imai T: Carbon-ion radiation enhances migration ability and invasiveness of the pancreatic cancer cell, PANC-1, in vitro. Cancer Sci 103: 677-683, 2012.

11 Fujita M, Yamada S and Imai T: Irradiation induces diverse changes in invasive potential in cancer cell lines. Semin Cancer Biol 35: 45-52, 2015.

12 Chikina AS and Alexandrova AY: An In Vitro System to Study the Mesenchymal-to-Amoeboid Transition. Methods Mol Biol 1749: 21-27, 2018

13 Kramer N, Walzl A, Unger C, Rosner M, Krupitza G, Hengstschläger $\mathrm{M}$ and Dolznig $\mathrm{H}$ : In vitro cell migration and invasion assays. Mutat Res 752: 10-24, 2013.

14 Alexandrova AY: Plasticity of tumor cell migration: acquisition of new properties or return to the past? Biochemistry (Mosc) 79: 947-963, 2014

15 Panková K, Rösel D, Novotný M and Brábek J: The molecular mechanisms of transition between mesenchymal and amoeboid invasiveness in tumor cells. Cell Mol Life Sci 67: 63-71, 2010.

16 Friedl P, Locker J, Sahai E and Segall JE: Classifying collective cancer cell invasion: Nat Cell Biol 14: 777-783, 2012.

17 Kim SH, Choe C, Shin YS, Jeon MJ, Choi SJ, Lee J, Bae GY, Cha HJ and Kim J: Human lung cancer-associated fibroblasts enhance motility of non-small cell lung cancer cells in coculture. Anticancer Res 33: 2001-2009, 2013.

18 Choe C, Shin YS, Kim SH, Jeon MJ, Choi SJ, Lee J and Kim J: Tumor-stromal interactions with direct cell contacts enhance motility of non-small cell lung cancer cells through the hedgehog signaling pathway. Anticancer Res 33: 3715-3723, 2013.

19 Ohuchida K, Mizumoto K, Murakami M, Qian LW, Sato N, Nagai E, Matsumoto K, Nakamura T and Tanaka M: Radiation to stromal fibroblasts increases invasiveness of pancreatic cancer cells through tumor-stromal interactions. Cancer Res 64: 32153212,2004 .
Received June 5, 2018

Revised June 21, 2018

Accepted June 26, 2018 